\title{
Group and case study of the dysexecutive syndrome in alcoholism without amnesia
}

\author{
H Ihara, G E Berrios, M London
}

\begin{abstract}
Objectives-To test the dysexecutive syndrome (DES) hypothesis of chronic alcoholism by the neuropsychological group and case study approaches.

Methods-A comprehensive neuropsychological assessment, including the "behavioural assessment of dysexecutive syndrome", a battery of tests recently designed to be "ecologically valid", was administered to 17 patients with chronic alcoholism without amnesia to examine executive functions, intelligence, and memory. In terms of each neuropsychological measure, reciprocal analyses of group means and individual case profiles were conducted: for the first contrasting the alcoholic patients with 17 age matched healthy subjects; and for the second making intersubject and intrasubject comparison of the patients, according to percentile basis impairment indices obtained from the control subjects.
\end{abstract}

Results-Despite relatively unimpaired memory and intelligence, the patients as a whole had the impairment of a wide range of executive domains, extending to "everyday" problem solving as well as more elementary aspects of executive functions, such as visuospatial performance, mental set shifting, and the inhibition of habitual behaviour. The profile analysis divided individual patients into four groups: the representative DES characterised by a clear dissociation between impaired executive functions and preserved intelligence and memory; the group of a modified dysexecutive pattern in which memory as well as executive functions were impaired with intelligence preserved; the group of general cognitive deterioration; and the group of unimpaired cognitive functioning. About two thirds of the patients were categorised into either the first or the second type of DES. Conclusion-DES characterised by the even more pronounced impairment of executive functions than of intelligence and memory afflicts a considerable proportion of patients with chronic alcoholism. Due to its subtlety, this would be potentially left out, unless appropriate behavioural measures were administered. This condition may prevent patients with alcoholism from achieving full recovery and benefiting from rehabilitation.

(F Neurol Neurosurg Psychiatry 2000;68:731-737)

Keywords: dysexecutive syndrome; executive functions; alcoholism; intelligence
Aspects of maladaptive behaviour in patients with alcohol dependence bear close resemblance to behaviour shown by subjects with lesions to the orbitofrontal cortex, thought to be responsible for inhibition of drives and affects. ${ }^{1}$ Such behaviour includes loss of control, inability to abstain from alcohol, repetitive habitual behaviour, and lack of forward planning. This behaviour is also analogous to what in neuropsychology is now called the dysexecutive syndrome (DES). ${ }^{2}$ The behavioural assessment of dysexecutive syndrome (BADS) has recently been devised to measure executive problems in daily living that are known to be an obstacle to treatment and rehabilitation. ${ }^{34}$

Interestingly, the neuropsychology of alcoholism without amnesia remains a less popular subject for study than that of alcoholism with amnesia, where both memory and executive functions have been intensively investigated. ${ }^{56}$ This is surprising, for only $10 \%$ of all alcoholic patients fulfil DSM-IV criteria for either alcohol induced persisting amnesic disorder or alcohol induced persisting dementia, ${ }^{67}$ which leaves the remaining $90 \%$ outside the scope of most neuropsychological studies. Although most alcoholic patients do not have cognitive impairment, ${ }^{89}$ they often achieve poor scores for performance IQ on the Wechsler adult intelligence scale (WAIS). ${ }^{810}$ These subtests require novel problem solving skills similar to those related to the executive tests, ${ }^{11}$ and results suggest the presence of subtle executive deficits in alcoholic patients. When more specific executive tests are applied, the finding is that alcoholic patients show poor performance on the trail making test part $\mathrm{B}^{9}{ }^{12-15}$; the Wisconsin card sorting test, ${ }^{16-18}$; and the Stroop test. ${ }^{15}$

This study was designed to test the hypothesis that latent DES may be an adverse factor in the rehabilitation of patients with chronic alcoholism. We considered the fact that alcoholic patients are likely to develop different cognitive patterns, due to factors such as variations in alcohol consumption and coexistence of drug misuse. Therefore, this study used a combination of group and individual case analyses of patients with alcoholism with and without opioid dependence. ${ }^{19-22}$

We focused on the extent and variation of the DES in non-amnesic alcoholic patients. We examined whether the patients as a whole showed impairment of executive functions despite heterogeneity and whether the neuropsychological deficit was disproportionate to the level of impairment shown in other higher cognitive functions. A detailed neuropsychological profile was provided to describe preserved abilities and cognitive deficits, 
according to percentile basis impairment for each measure obtained from 17 age matched control subjects.

\section{Subjects and methods \\ SUBJECTS}

Seventeen patients (13 men and four women), recruited from the Drug and Alcohol Service, Brookfield Hospital, Cambridge, participated in this study. We excluded those who were aged over 55, those who had been previously diagnosed as having Korsakoff's syndrome, those who had a history of head injury with loss of consciousness, and those who had any comorbidity with other mental, neurological, or systematic illnesses. They were alcohol free (breathalyser) and detoxified from alcohol for at least 3 weeks (self report). Thirteen of the patients were taking medication at the time of the neuropsychological assessment, including antabuse $(n=2)$, diazepam $(n=4)$, lithium $(n=1)$, prozac $(n=2)$, chlomipramine $(n=1)$, and carbamazepine $(n=1)$. All patients met the criteria for alcohol dependence, but not for either alcohol induced persisting amnesic disorder or alcohol induced persisting dementia, according to DSM-IV. ${ }^{7}$ All patients underwent a semistructured diagnostic interview for the DSM-IV that included questions about alcohol related problems of memory and concentration even when not drinking. Detailed examination of medical records also showed that none of the alcoholic patients had a history of Wernicke's encephalopathy, acute confusion, severe anterograde amnesia, retrograde amnesia, or temporal and spatial disorientation related to alcohol; nor did they show impaired social or occupational functioning or present a significant deficit from a previous level of functioning. Ten patients met criteria for opioid dependence, and five were taking methadone. The five opioid dependent patients not taking methadone were abstinent from opioids at the time of testing. To estimate premorbid intelligence, we applied the national adult reading test (NART) to all patients. ${ }^{23}$ For the assessment of addictive behaviour, we asked all patients to fill out the self administered short Michigan alcoholism screening test (SMAST) and its reworded version for drug use (SMAST-D). ${ }^{24}$ Basic variables in this group were as follows (means (SD)): age 38.3 (9.0 years; duration of education: 11.9 (3.3) years; duration of dependence 19.1 (10.4) years; NART IQ 108.4 (13.8); SMAST score 36.3 (12.8); and SMAST-D score 21.6 (18.5).

CONTROLS

Seventeen control subjects (two men and 15 women) responded to advertisements for paid volunteers at the Medical Research Council Cognition and Brain Sciences Unit, Cambridge and cooperated in obtaining norms of each neuropsychological test. We excluded those who were aged over 57, those who had a family history of alcoholism, those who had any history of substance misuse including alcohol dependence, those who had any history of head injury with loss of consciousness, and those who had any comorbidity with other mental, neurological, or systematic illnesses. The control subjects were matched with the patients with alcoholism for age (mean 41.8 (SD 10.6) and NART based estimated premorbid intelligence (mean 103.5 (SD 6.1).

Ethical approval for the study was obtained from the Cambridge local research ethics committee and all patients and control subjects signed a consent form.

NEUROPSYCHOLOGICAL TEST BATTERY

All patients and control subjects were given two intelligence tests - the NART and the Raven advanced progressive matrices (RAPM). The first is a test of premorbid intelligence, and the second one of current intelligence. An extended battery of neuropsychological tests was then applied to all subjects, and included: the signal detection recognition memory test $(\text { SIGNAL) })^{25}$; the cognitive estimation test $(\mathrm{CET})^{26}$; the verbal fluency test $(\mathrm{VFT})^{27}$; the trail making test $(\mathrm{TMT})^{28}$; the modified Wisconsin card sorting test $\left(\mathrm{MCST}^{29}\right.$; the Stroop colour word test $(\mathrm{SCW})^{30}$; the goal neglect test $(\mathrm{GNT})^{31}$; and the behavioural assessment of dysexecutive syndrome (BADS). ${ }^{3}$ A thumbnail sketch of the SIGNAL and the BADS are provided here. Detailed descriptions of the tests can be found in the literature shown above. All patients were examined by a psychiatrist (HI), who did not know which group patients belonged to at the time of testing.

\section{Signal detection recognition memory test $(S I G N A L)^{25}$}

This computerised recognition memory test in "yes-no" form comprises 120 words. Based on the false alarm rate and the hit rate of the subject, $d^{\prime}$ ' is obtained (from table of $d^{\prime}$ ' and beta). ${ }^{32}$ According to the theory of signal detection recognition memory, the measure of discriminability $\left(d^{\prime}\right)$ reflects the sensitivity of "pure" memory processes. We routinely adopted Snodgrass and Corwin's ${ }^{33}$ method of correction, regardless of the presence or absence of 1.0 hit rates and 0 false alarm rates.

\section{Behavioural assessment of dysexecutive syndrome} $(B A D S)^{3}$

This consists of six subtests, for each of which a summary profile score is obtained (with a maximum of 4 and minimum of 0 ). These are then added up to produce an overall score (out of 24).

(1) Rule shift cards - In trial 1 of this test the subject is asked to say "yes" to a red card and "no" to a black card, while 21 spiral bound non-court playing cards are turned over one by one. In trial 2 the subject is asked to say "Yes if the card is the same colour" as the previous one, otherwise to say "No".

(2) Action programme-The subject is given a rectangular stand on which a beaker and a tube are placed, the first full of water and covered by a removable lid with a small hole in it and the second with a small piece of cork at its bottom. The subject is asked to get the cork out of the tube by using any of the following: a metal, L shaped rod which is too short to reach 
Table 1 Means scores (SD) of the alcoholic patients and the healthy control subjects and the Mann-Whitney $U$ test results between the two groups on the RAPM and the SIGNAL

\begin{tabular}{|c|c|c|c|c|c|}
\hline & \multirow[b]{2}{*}{$R A P M$} & \multicolumn{4}{|l|}{ SIGNAL } \\
\hline & & Corrected hit rate & $\begin{array}{l}\text { Corrected false } \\
\text { alarm rate }\end{array}$ & $d^{\prime}$ & Beta \\
\hline Alcoholic patients & $7.71(2.39)$ & $68.1(27.1)$ & $12.7(12.8)$ & $1.91(0.89)$ & $2.58(2.58)$ \\
\hline Controls & $9.18(2.21)$ & $85.0(12.7)$ & $10.7(7.2)$ & $2.58(0.48)$ & $2.43(2.71)$ \\
\hline $\begin{array}{l}\text { Mann-Whitney } U \text { between alcoholic } \\
\text { patients and controls } \\
\text { Asymp significance }(2 \text { tailed })\end{array}$ & $\begin{array}{l}U=100.5 \\
\mathrm{p}=0.126\end{array}$ & & & $\begin{array}{l}U=72.0 \\
\mathrm{p}=0.130\end{array}$ & \\
\hline
\end{tabular}

RAPM=Raven advanced progressive matrices $\left(\right.$ Raven $\left.^{34}\right)$; SIGNAL=signal detection recognition memory test $\left(\right.$ Banks $\left.^{25}\right)$

the cork; a small screw top container, and its unscrewed top. A stipulation is that the subject is forbidden to lift up the stand, the tube or the beaker, or to touch the lid with his or her fingers.

(3) Key search- $-\mathrm{He}$ or she is asked to imagine that a $100 \mathrm{~mm}$ square drawn in the centre of a piece of paper is a large field and that somewhere in this field he or she has lost his or her keys. The task is to draw a line to show how the keys can be found in the field.

(4) Temporal judgement-This test comprises four questions which require the subjects to estimate the time needed for an activity.

(5) Zoo map-This test consists of two parts, both of which ask subjects to show how they would reach designated places in a zoo, using a map without breaking the rule of using designated paths once only. The first part requires the subject to plan the order in which to visit locations. The second part gives the subject explicit instructions on how to plan a route.

(6) Modified six elements test-The subject is set three tasks - dictation, arithmetic, and picture naming - each of which consists of two parts, hence six subtasks in total. The subject has to do at least something from each of the six subtasks within 10 minutes. Besides, the subject must not do two parts of the same task one after the other.

ANALYSES

We used the SPSS V7 package for descriptive and inferential statistical analyses. The latter was the Mann-Whitney $U$ test for comparison of performances by alcoholic patients with those of the controls on the neuropsychological tests. For the intergroup comparison, we adopted non-parametric analysis, instead of the $t$ test, because some of the scores on the tests, such as the GNT and the BADS subtests, were not distributed normally.

\section{Results}

COMPARISON BETWEEN GROUPS

Table 1 shows that the Mann-Whitney $U$ test did not find statistically significant differences between alcoholic patients and controls on current intelligence and recognition memory, although the above point-such group statistics may conceal subtle intergroup differencestill applies. Be that as it may, these results can be interpreted cautiously as suggesting that the alcoholic patients of our sample did not show marked intellectual or memory disorders.

Tables 2 and 3 show results for five traditional executive tests; the GNT, a computerised letter monitoring test; and the BADS. Analysis using non-parametric statistics showed significant differences between alcoholic patients and controls on the BADS as well as on the TMT, the MCST, and the SCW. As far as we know, this is the first time that an impaired performance on the BADS has been shown in relation to chronic alcoholism. Two of the six subtests of the BADS, the temporal judgement and the modified six elements tests, were particularly sensitive to alcohol related executive dysfunction. Our finding that alcoholic patients perform poorly on the GNT replicates a similar finding for frontal patients. ${ }^{31}$ Earlier findings were also confirmed by our results of a low performance by alcoholic patients on the TMT Part B, ${ }^{9}{ }^{12-15}$ the Wisconsin card sorting test, ${ }^{16-18}$ and the SCW. ${ }^{15}$ Intergroup differences between the alcoholic patients and the controls on the VFT and the CET did not reach significance.

In addition, we tried the Mann-Whitney $U$ tests to distinguish between the opioid dependent and non-opioid dependent subgroups, but did not find any significant intergroup differences for cognitive measures. Moreover, we failed to find any significant correlations and partial correlations between performances on the cognitive measures and duration of alcohol-

Table 2 Means scores (SD) of the alcoholic patients and the healthy control subjects and the Mann-Whitney U test results between the two groups on the VFT, the TMT, the MCST, the SCW, and the GNT

\begin{tabular}{|c|c|c|c|c|c|c|}
\hline & $V F T$ & $T M T: B-A$ & $C E T$ & $\begin{array}{l}\text { MCST: categories } \\
\text { achieved }\end{array}$ & $S C W$ & $\begin{array}{l}\text { GNT: passed switch } \\
\text { trials }\end{array}$ \\
\hline Alcoholic patients & $40.5(16.0)$ & $74.5(75.2)$ & $5.94(4.24)$ & $4.38(1.89)$ & $89.9(24.9)$ & $3.92(2.36)$ \\
\hline Controls & $46.4(15.7)$ & $30.5(14.7)$ & $4.06(2.75)$ & $5.82(0.39)$ & $107.4(8.39)$ & $5.59(0.62)$ \\
\hline $\begin{array}{l}\text { Mann-Whitney } U \text { between alcoholic } \\
\text { patients and controls }\end{array}$ & 122.5 & 76.0 & 108.0 & 73.0 & 77.0 & 65.5 \\
\hline Asymp significance (2 tailed) & 0.448 & $0.018^{\star}$ & 0.204 & $0.008^{\star \star}$ & $0.014^{\star}$ & $0.039^{\star}$ \\
\hline
\end{tabular}

${ }^{\star} \mathrm{p}<0.05 ;{ }^{\star \star} \mathrm{p}<0.01$, Mann-Whitney $U$ test.

VFT=Verbal fluency test $\left(\right.$ Miller $\left.^{27}\right)\left(\mathrm{F}, \mathrm{A}, \mathrm{S}\right.$ total words); TMT $=$ trail making test $\left(\operatorname{Reitan}^{28}\right)$ (time taken for part A subtracted from that for part $\left.\mathrm{B}\right) ; \mathrm{CET}=$ cognitive estimates test $\left(\right.$ Shallice and Evan $\left.{ }^{26}\right) ; \mathrm{MCST}=$ modified card sorting test $\left(\right.$ Nelson $\left.{ }^{29}\right)\left(\right.$ number of categories achieved); SCW $=\mathrm{Stroop}$ test $\left(\right.$ Trenerry $e t$ a $\left.{ }^{30}\right)($ number of ink colours achieved); GNT=goal neglect test (Duncan et $a l^{\beta 1}$ ) (number of passed switch trials). 
Table 3 Mean scores (SD) of the alcoholic patients and the healthy control subjects and the Mann-Whitney $U$ test results between the two groups on the $B A D S$ subtests and total BADS score

\begin{tabular}{|c|c|c|c|c|c|c|c|}
\hline & Rule shift cards & $\begin{array}{l}\text { Action } \\
\text { programme }\end{array}$ & Key search & Time judgement & Zoo map & $\begin{array}{l}\text { Modified six } \\
\text { elements }\end{array}$ & $B A D S$ total score \\
\hline Alcoholic patients & $3.47(1.07)$ & $3.59(1.00)$ & $3.24(1.15)$ & $1.59(1.00)$ & $2.71(1.21)$ & $3.24(1.20)$ & $17.82(4.39)$ \\
\hline Controls & $3.82(0.52)$ & $3.82(0.39)$ & $3.53(0.72)$ & $2.65(0.61)$ & $3.47(0.62)$ & $4.00(0.00)$ & $21.29(1.45)$ \\
\hline Mann-Whitney $U$ between alcoholic & & & & & & & \\
\hline patients and controls & 119.0 & 141.5 & 130.0 & 59.5 & 95.0 & 85.0 & 68.5 \\
\hline Asymp sig (2 tailed) & 0.213 & 0.876 & 0.565 & $0.002^{\star \star}$ & 0.068 & $0.004^{\star \star}$ & $0.008^{\star \star}$ \\
\hline
\end{tabular}

${ }^{\star \star} \mathrm{p}<0.01 ;$ Mann-Whitney $U$ test.

BADS=Behavioural assessment of dysexecutive syndrome (Wilson et al ${ }^{\beta}$ ).

ism in the patient group, irrespective of the presence and severity of opioid dependence.

INDIVIDUAL CASE ANALYSIS OF ALCOHOLISM

Table 4 gives background information on individual patients and table 5 lists neuropsychological test results for all patients. One patient refused to perform the MCST and four patients did not perform the GNT. The level of impairment per subject was calculated according to the following index: percentile ranges of the data from the 17 control subjects were divided into four bands: $A>34$ th percentile; $\mathrm{B}=12-34$ th percentile; $\mathrm{C}=5-11$ th percentile; and $\mathrm{D}<5$ th percentile. In addition, we make a single division into normal (index $\mathrm{A}$ or $\mathrm{B}$ ) or impaired (index $\mathrm{C}$ or $\mathrm{D}$ ) in each individual test. The advantage of this method is that a common grading, based on the score of the same control group, can be applied to all the neuropsychological test scores of each patient.

This is particularly apt for the purpose of interindividual and intraindividual comparisons of raw data. Shallice et $a l^{20}$ used a similar method in their case study of schizophrenia, although their impairment index was not standardised in terms of the same control subjects performing on different neuropsychological tests.

Alcoholism without opioid dependence

Six of the seven patients with alcoholism and without opioid dependence performed poorly on at least one of the major executive tests. Three patients (A5, A6, A7) showed a marked discrepancy between their performances on the executive tests and their estimated premorbid

Table 4 Demographic and background information of the individual patients with alcoholism

\begin{tabular}{lllllllll}
\hline & Fincation & $\begin{array}{l}\text { Duration of } \\
\text { dependence }\end{array}$ & $\begin{array}{l}\text { Alcoholism } \\
\text { (yes: no) }\end{array}$ & $\begin{array}{l}\text { Opioid } \\
\text { dependence } \\
\text { (yes: no) }\end{array}$ & SMAST & SMAST-D \\
\hline A1 & M & 54 & 11 & 40 & Yes & No & 42 & 0 \\
A2 & M & 38 & 9 & 2 & Yes & No & 50 & 0 \\
A3 & M & 38 & 11 & 19 & Yes & No & 51 & 0 \\
A4 & M & 32 & 11 & 18 & Yes & No & 36 & 6 \\
A5 & M & 35 & 10 & 18 & Yes & No & 47 & 4 \\
A6 & M & 31 & 17 & 8 & Yes & No & 28 & 1 \\
A7 & M & 51 & 16 & 17 & Yes & No & 29 & 0 \\
A8 & F & 27 & 16 & 9 & Yes & Yes & 33 & 26 \\
A9 & F & 33 & 10 & 16 & Yes & Yes & 42 & 45 \\
A10 & M & 32 & 11 & 14 & Yes & Yes & 18 & 50 \\
A11 & M & 51 & 11 & 34 & Yes & Yes & 10 & 25 \\
A12 & M & 35 & 10 & 18 & Yes & Yes & 45 & 31 \\
A13 & M & 52 & 9 & 38 & Yes & Yes & 47 & 40 \\
A14 & M & 47 & 17 & 29 & Yes & Yes & 21 & 31 \\
A15 & M & 31 & 16 & 15 & Yes & Yes & 44 & 43 \\
A16 & M & 29 & 11 & 12 & Yes & Yes & 51 & 35 \\
A17 & M & 35 & 6 & 18 & Yes & Yes & 23 & 31 \\
\hline
\end{tabular}

SMAST $=$ Self administered short Michigan alcoholism screening test (Selzer et $\left.a l^{4}\right)$; SMAST-D = self administered short Michigan alcoholism screening test reworded for drug misuse (Selzer $e t$ $a l^{24}$. intelligence as indicated by NART IQ and education and occupation histories. A6 showed disproportionate executive deficits in the face of intact recognition memory. A4, on the other hand, also had memory impairment. Two patients (A1 and A2) showed not only an impairment in executive functions but also a general intellectual deterioration and a reduced NART score, leaving unresolved the question of whether their overall cognitive deficits were attributable to DES or to premorbid low functioning. A3 was unimpaired in all cognitive domains despite his long term alcoholism.

\section{Alcoholism with opioid dependence}

All but two (A8, A12) of 10 alcoholic patients with opioid dependence showed signs of DES. Seven (A9, A10, A11, A13, A14, A16, A17) showed a marked discrepancy between their relatively high scores on the current intelligence test and their poor performance on executive tests. On the other hand, A 15 showed an intellectual decline, indicated by the contrast between his high score on the NART and the poor score on the RAPM. Four patients (A9, A10, A11, A13) performed poorly on the memory test as well as on the executive tests, albeit performing well on the RAPM intelligence test. Two patients (A8, A12) showed few signs of DES.

\section{Example of DES case}

Patient A5-This patient had a long history of alcoholism, with four emergency admissions to hospital due to acute intoxication. Performance on the two baseline tests indicated average intelligence. Performance on the VFT was poorer than predicted by his NART IQ; he only produced seven words starting with the letter A, none of which was expressed in the second half of the 60 second period. In the MCST, he completed the first three categories without any mistakes, by striking contrast with 18 subsequent errors made before finishing a fourth category. After finishing the colour task section of the Stroop test in only 1 minute, he performed significantly more slowly on the colour-word task, completing only 94 items in 2 minutes. He failed in three out of six stay trials and in two out of six switch trials on the GNT, showing typical goal neglect in the second. It is worth mentioning that he succeeded on the first switch trial, followed by repeated goal neglect performances. In the action programme of the BADS he could not relate objects to each other, although he glanced at each of them. He could not complete three of the five stages necessary for this task - namely, removing the lid from the 
Table 5 Test results of the individual patients with alcoholism

\begin{tabular}{|c|c|c|c|c|c|c|c|c|c|c|c|c|c|c|c|c|}
\hline & $N A R T I Q$ & $R A P M$ & $\begin{array}{l}\text { SIGNAL } \\
\left(d^{\prime}\right)\end{array}$ & $V F T$ & $T M T$ & $C E T$ & $M C S T$ & $S C W$ & $G N T$ & $\begin{array}{l}\text { Rule shift } \\
\text { cards }\end{array}$ & $\begin{array}{l}\text { Action } \\
\text { program }\end{array}$ & $\begin{array}{l}\text { Key } \\
\text { search }\end{array}$ & $\begin{array}{l}\text { Temporal } \\
\text { judgement }\end{array}$ & $\begin{array}{l}\text { Zoo } \\
\text { map }\end{array}$ & $\begin{array}{l}\text { Modified six } \\
\text { elements }\end{array}$ & $\begin{array}{l}\text { BADS } \\
\text { total }\end{array}$ \\
\hline $\mathrm{A} 1$ & 76 & D & D & D & D & C & D & $\mathrm{D}$ & & C & A & A & C & C & $\mathrm{D}$ & D \\
\hline A2 & 87 & D & D & B & B & D & D & B & A & A & A & A & C & A & A & A \\
\hline A3 & 108 & A & A & A & A & A & $\mathrm{A}$ & A & B & A & A & A & C & A & A & A \\
\hline A4 & 110 & A & D & A & A & B & B & A & A & A & A & B & D & C & A & D \\
\hline A5 & 107 & B & $\mathrm{D}$ & B & B & A & $\mathrm{D}$ & C & C & A & D & B & A & $\mathrm{C}$ & A & $\mathrm{D}$ \\
\hline A6 & 124 & A & A & A & B & A & A & B & $\mathrm{D}$ & A & A & A & B & A & $\mathrm{D}$ & A \\
\hline A7 7 & 95 & D & D & D & D & C & D & D & & D & A & D & C & D & D & D \\
\hline A8 & 123 & A & $\mathrm{D}$ & A & A & A & A & $\mathrm{A}$ & A & A & A & $\mathrm{A}$ & B & A & A & A \\
\hline A9 & 118 & A & D & C & D & D & & $\mathrm{D}$ & D & A & A & C & A & A & A & B \\
\hline A 10 & 98 & A & $\mathrm{C}$ & A & $\mathrm{C}$ & A & A & $\mathrm{D}$ & $\mathrm{C}$ & A & A & A & A & A & A & A \\
\hline A11 & 116 & B & $\mathrm{D}$ & A & D & A & A & $\mathrm{D}$ & & A & A & C & D & C & $\mathrm{D}$ & $\mathrm{D}$ \\
\hline A 12 & 116 & A & B & A & A & B & A & B & A & A & A & A & C & A & A & A \\
\hline A13 & 106 & A & $\mathrm{D}$ & A & $\mathrm{D}$ & A & B & $\mathrm{D}$ & & A & $\mathrm{D}$ & A & B & $\mathrm{D}$ & $\mathrm{D}$ & $\mathrm{D}$ \\
\hline A14 & 123 & B & B & A & C & A & D & A & B & A & A & A & A & $\mathrm{C}$ & A & A \\
\hline A15 & 121 & C & A & A & A & A & $\mathrm{D}$ & C & $\mathrm{D}$ & C & A & C & C & $\mathrm{D}$ & $\mathrm{D}$ & $\mathrm{D}$ \\
\hline A16 & 117 & A & A & A & $\mathrm{D}$ & C & $\mathrm{A}$ & C & A & C & A & A & B & A & A & A \\
\hline A17 & 97 & A & A & C & B & B & $\mathrm{D}$ & A & D & C & B & $\mathrm{B}$ & C & C & $\mathrm{D}$ & D \\
\hline
\end{tabular}

A $>4$ th percentile; $B=12-34$ th percentile; $C=5-11$ th percentile; $\mathrm{D}<5$ th percentile.

NART $=$ National adult reading test; RAPM=Raven advanced progressive matrices; SIGNAL ( $d^{\prime}$ )=discriminability in the signal detection recognition memory test; VFT=verbal fluency test; TMT=time taken for part B subtracted from the time take for part A in the trail making test; CET=cognitive estimates test; $M C S T=$ modified card sorting Test; SCW=colour-word task of the Stroop test; GNTpsw=passed switch trials of the goal neglect test; BADS total=total score on the behavioural assessment of dysexecutive syndrome.

beaker using the wire hook, attaching the screw top to the container, and filling the container with water. On the zoo map test version 1 he showed himself to be a recklessly good loser. Firstly, he drew a line to indicate visits to a few designated places. As soon as he found this was a wrong way, however, he abandoned all efforts to continue the task. He immediately accepted his failure with resignation, and the examiner's encouraging explanation that this was not a timing test did not prompt him to try again.

\section{Discussion}

Our results indicate that patients with chronic alcoholism show mild but significant DES even in the presence of relatively unimpaired intelligence and memory. Due to its subtlety, DES runs the risk of remaining undetected, unless appropriate neuropsychological measures are administered. These performance deficits are not explicable in terms of the concurrent misuse of opioids, as lower executive levels are found even in patients without a history of opioids misuse. It seems unlikely that the poor performance on executive tests in our study is due to drug treatment. Indeed, about two thirds of the alcoholic patients were taking medication at the time of testing. There was, however, no obvious relation between test performance and dose level. Besides, definite DES was found even in patients not on medication, such as A5 and A7. At the same time, analysis of individual cases confirms marked interindividual and intraindividual differences in cognitive performances. Such a heterogeneity reflects not only on the presence or absence of DES, but also on the severity and pattern of executive deficits, and of concomitant disorders of intelligence and memory.

The disproportionate impairment of executive functions in relation to generalised intellectual decline cannot be attributed to a limited sensitivity of the RAPM. The RAPM ${ }^{34}$ requires the subject to perceive and discriminate spatial, design, and numerical relations. Like Cattell's culture fair, ${ }^{35}$ this test is intended to be a knowledge free test of general ability, because it requires neither language nor academic skills for success, but, rather a capacity for novel problem solving. In this respect, the RAPM shares with Cattell's culture fair the characteristics of "fluid intelligence" tests. On the other hand, the WAIS in general and its verbal subtests in particular involve mainly "crystallised" ability, which refers to well established habits or skills of perception and behaviour in routine circumstances. ${ }^{36}$ The WAIS should, accordingly, reflect long stored information and well practised skills, which are resistant to subsequent brain damage. Therefore, the RAPM as a method of assessing fluid ability and current intelligence is superior to the WAIS. Nevertheless, the RAPM did not register significantly low intelligence in the patients with alcoholism in this research.

A profile analysis of 17 patients with alcoholism shows that in two thirds of these patients executive function impairment is independent of general intelligence. Higher cognitive functioning in patients with alcoholism shows roughly four patterns (described below), two of which are characterised by impaired executive functions and preserved intelligence. The first pattern is a typical DES, characterised by a clear dissociation between impaired executive functions and preserved memory and intelligence. ${ }^{40}$ This applies to patients A6, A14, A16, and A17, and probably to A15. Some of the patients in this group can also be considered to be in the process of undergoing a change from a typical DES to a more general cognitive disorder, including memory and intelligence. For example, A14 presented low intelligence, in comparison with estimated premorbid level, although his current intelligence remained statistically normal. A15 also achieved a lower score on the current intelligence test, relative to the estimated premorbid level indicated by his educational history and NART IQ.

The second pattern consists of a modified dysexecutive syndrome with impaired memory and a normal level of intelligence. Patients A4, A5, A9, A10, A11, and A13 showed this pattern. The third pattern was characterised by a general cognitive deterioration affecting both 
intelligence and memory as well as executive functions, and relating to A1, A2, and A7. The fourth pattern consists of patients who showed no signs of cognitive impairment (A3 and A12 and probably A8, despite her poor performance on the SIGNAL). The poor result of A8 on this test could possibly be attributed to impulsive decision making, for the following reason; her false alarm rate was as high as $46.25 \%$ - that is, in $37 / 80$ she mistook noise for a signal, whereas her hit rate was reasonably high (only two missing out of 40 signals).

The functional independence of executive functions from memory in patients with alcoholism leaves room for further investigation. Indeed, we excluded patients with alcohol induced amnesic disorder in the initial interview. Psychiatric diagnosis is mostly based on descriptive information provided by the patient, ${ }^{7}$ and relies on the assumption that the patient is aware of his or her own mental problems and is able to verbalise them with reasonable accuracy. The fact that 10 out of 17 alcoholic patients perform poorly on the signal detection recognition memory test casts doubt on this assumption. However, the decision as to whether a patient has a memory problem should be based on several different memory tests. Consequently we do not want to speculate further on the relation between executive functions and memory in alcoholism. Furthermore, a normal performance on memory tests does not guarantee absence of alcohol related brain damage and hence additional executive tests are needed to test whether maladaptive alcohol related problems are associated with brain dysfunction.

We should emphasise the fact that statistically significant results in executive measures were found despite the existence of qualitatively different forms of impairment in alcoholic patients. Patients showed marked clumsiness when faced with a task that requires not only speed of response but also cognitive flexibility. For example, they performed poorly on both the TMT and the SCW, which examine the subject's cognitive flexibility, as well as mere speed of response. On the TMT the subject must shift his or her mental set between alphabetical and numerical order, and on the SCW the subject must read printed colour names while inhibiting the conflicting shape of the word as a predominant stimulus. By contrast, we found the VFT to be a rather blunt instrument for the assessment of DES in chronic alcoholism. Although this test is thought to be sensitive to frontal executive dysfunction, the task involved in this test is the initiation and continuation of relatively simple verbal acts. These findings imply that inertia in alcoholic patients is not due to the limited facility with which they can produce variable behaviour. Rather, it is due to the difficulty of changing mental sets and integrating behavioural fragments into a coherent stream of conduct.

Results on the BADS show that cognitive deficits in patients with alcoholism extend to everyday executive performances as well as to more elementary aspects of executive func- tions, such as psychomotor speed, mental flexibility, and inhibitory control over impulsive responses. However, in our sample, performance on the BADS did not always predict normal or impaired performance on other executive tests. For example, among nine normal performers on the BADS, four achieved poor scores on the TMT, two on the MCST, three on the SCW, and three on the GNT. Therefore, we should consider the results for other measures as well as those for the BADS for a cognitive assessment of individual alcoholic patients.

Among six subtests of the BADS we found the modified six elements useful as it highlights more than other subtests aspects of poor planning in alcoholism. For the purpose of avoiding a breach of the rules governing the experiment, most control subjects in our sample figured out a few devices, such as working out an appropriate sequence before they started the test to monitor the progress of their performance, making a note of a completed subtest before switching from it to another, and so on. By contrast, alcoholic patients neither contrived such a means beforehand, nor made any effort to do so while performing the test. Some patients took excessive time for one particular subtask. Other patients shifted subtasks every few seconds, stopped after completing each of the six subtasks, retried each of them again and again, and eventually broke the rule governing the test. Such negligence of rules and poor planning in alcoholic patients contrast sharply with the fact that they could verbalise the rules both before and after their performances.

The biological bases for low scoring on executive tests and aberrant test behaviour in alcoholic patients are of interest. Recent work shows that, regardless of the presence or absence of memory disorder, alcoholic patients have a loss of frontal lobe volume at the expense of white matter, and a selective neuronal loss in the superior frontal association cortex, with motor cortex spared. ${ }^{36}$ Findings supporting frontal pathology are also reported by an MRI study ${ }^{36}$ and PET studies. ${ }^{18}{ }^{37}$ This is convergent with our neuropsychological DES findings in chronic alcoholism.

These findings are also relevant to treatment and rehabilitation. Aspects of alcohol related behaviour, such as unsuccessful abstinence and lack of forward thinking, have often been the subject of predominantly psychodynamic interpretations. ${ }^{14}$ There is, however, a possibility that lack of staying power in alcoholic patients may also be related to DES. In fact, the results of this study suggest that alcoholic patients have difficulty when demonstrating abstract analysis, critical judgement, and flexibility of thought process. As a result, their ability to respond to conventional alcohol rehabilitation programmes, such as educational information presented in lecture form, verbally mediated group sessions, and individual psychotherapy may be compromised. If DES precludes alcoholic patients from learning, then these didactic efforts may not work, and outcomes will be poor. 


\section{Conclusions}

To test the DES hypothesis of chronic alcoholism, a comprehensive neuropsychological assessment, including the "behavioural assessment of dysexecutive syndrome", was applied to 17 patients with chronic alcoholism and without amnesic syndrome. Despite their relatively preserved memory and intelligence, these patients were found to be impaired on a wide range of executive domains, including that of everyday problem solving. A profile analysis based on percentile calculations showed that about two thirds of the patients fell into the DES category with pronounced impairment of executive functions despite preserved memory and intelligence. This suggests that the management of patients with chronic alcoholism requires a more detailed neuropsychological profiling than is currently in use.

We thank all staff members of Drug and Alcohol Service, Brookfield Hospital who allowed us to examine patients under their care. We have received no external funding for this research. This work was performed by Hiroshi Ihara as part of the PhD dissertation submitted to the Department of Psychiatry, the University of Cambridge, 2000.

1 Fuster JM. The prefrontal cortex: anatomy, physiology, and neuropsychology of the frontal lobe. 2nd ed. New York: Raven ropsychology

2 Baddeley AD. Working memory. Oxford: Oxford University Press, 1986

3 Wilson BA, Alderman N, Burgess P, et al. Behavioural assessment of the dysexecutive syndrome (BADS). Bury St Edmunds: Thames Valley Test Company, 1996.

4 Evans JJ, Chua SE, McKenna PJ, et al. Assessment of the dysexecutive syndrome in schizophrenia. Psychol Med 1997;27:635-46.

5 Jacobson RR, Lishman WA. Selective memory loss and global intellectual deficits in alcoholic Korsakoff's syndrome. Psychol Med 1987;17:649-55.

6 Rourke SB, Løberg T. The neurohehavioral correlates of alcoholism. In: Grant I, Adams KM, eds. Neuropsychological assessment of neuropsychiatric disorders. Oxford; Oxford University Press, 1996:423-85.

7 American Psychiatric Association. Diagnostic and statistical manual of mental disorders. 4th ed. Washington, DC: APA, 1994.

8 Fitzhugh LC, Fitzhugh KB, Reitan RM. Adaptive abilities and intellectual functioning in hospitalised alcoholics. Quarterly fournal of Studies on Alcohol 1960;21:414-23.

9 Kleinknecht RA, Goldstein SG. Neuropsychological defiicts associated with alcohol. A review and discussion. Quarterly fournal of Studies on Alcohol 1972;33:999-1019.

10 Jonsson CO, Cronholm B, Izikowitz S. Intellectual changes in alcoholics. Quarterly fournal of Studies on Alcoho 1962;23:221-42.

11 Walsh KW. Understanding brain damage: a primer of neuropsychological evaluation. 2nd ed. London: Churchil Livingstone, 1991.
12 Goldstein G, Shelly C. Field dependence perceptual and motor skills in alcoholics: a factor analytic study. Quarterly fournal of Studies on Alcohol 1971;32:29-40.

13 Løberg T. Alcohol misuse and neuropsychological deficits in men. Fournal of Studies on Alcohol 1980;41:119-28.

14 Gordon SM, Kennedy BP, McPeake JD. Neuropsychologically impaired alcoholics: assessment, treatment considerations, and rehabilitation. I Subst Abuse Treat 1988;5:99104.

15 Goldstein G, Chotlos J. Dependency and brain damage in alcoholics. Percept Mot Skills 1965;21:135-50.

16 Tarter RE. An analysis of cognitive deficits in chronic alcoholics. F Nerv Ment Dis 1973;157:138-47.

17 Tarter RE, Parsons OA. Conceptual shifting in chronic alcoholics. F Abnorm Psychol 1971;77:71-5.

18 Adams KM, Gilman S, Koeppe RA, et al. Neuropsychological deficits are correlated with frontal hypometablism in positron emission tomography studies of older alcoholic patients. Alcohol Clin Exp Res 1993;17:205-10.

19 Caramazza A. On drawing inferences about the structure of normal cognitive systems from the analysis of patterns of impaired performance: the case for single-patient studies. Brain Cogn 1986;5:41-66.

20 Shallice T, Burgess PW, Frith CD. Can the neuropsychological case-study approach be applied to schizophrenia? Psychol Med 1991;21:661-73.

21 Walsh KW. Neuropsychology: a clinical approach. 2nd ed. Edinburgh: Churchill Livingstone, 1987.

$22 \mathrm{McFie}$ J. Psychological testing in clinical neurology. $7 \mathrm{Nerv}$ Ment Dis 1960;131:383-93.

23 Nelson $\mathrm{H}$, Willison JR. The national adult reading test (NART). 2nd ed. Windsor: NFER-Nelson, 1991

24 Selzer ML, Winokur A, van Rooijen L. A self-administered short alcoholism screening test (SMAST). Fournal of Studies on Alcohol 1975;36:117-26.

25 Banks WP. Signal detection theory and memory. Psychol Bull 1970;74:81-99.

26 Shallice T, Evans ME. The involvement of the frontal lobes in cognitive estimation. Cortex 1978;14:294-303.

27 Miller E. Verbal fluency as a function of a measure of verbal intelligence and in relation to different types of cerebral pathology. Br f Clin Psychol 1984;23:53-7.

28 Reitan RM. Validity of the trail-making test as an indication of organic brain damage. Percept Mot Skills 1958;8:271-6.

29 Nelson HE. A modified card sorting task sensitive to frontal lobe defects. Cortex 1976;12:313-24.

30 Trenerry MR, Crosson B, DeBoe J, et al. Stoop Neuropsychological screening test manual. Odessa, FL: Psychological Assessment Resources, 1989.

31 Duncan J, Emslie H, Williams P, et al. Intelligence and the frontal lobe: the organization of goal-directed behavio Cognitive Psychology 1996;30:257-303.

32 Freeman PR. Table of d' and $\beta$. Cambridge: Cambridge University Press, 1973.

33 Snodgrass JG, Corwin J. Pragmatics of measuring recognition memory: applications to dementia and amnesia. $\mathcal{f}$ Exp Psychol Gen 1988;117:34-50.

34 Raven JC. Advanced progressive matrices. Oxford; Oxford Psychologists, 1976

35 Cattell RB, Cattell AKS. Test of ' $g$ ': culture fair. Champaign, IL: Institute for Personality and Ability Testing, 1973.

36 Pfefferbaum A, Sullivan EV, Mathalon DH, et al. Frontal lobe volume loss observed with magnetic resonance imaging in older chronic alcoholics. Alcohol Clin Exp Res 1997;21:521-9.

37 Johnson-Greene D, Adams KM, Gilman S, et al. Effects of abstinence and relapse upon neuropsychological function and cerebral glucose metabolism in severe chronic alcoholism. f Clin Exp Neuropsychol 1997;19:378-85. 